Revista Interdisciplinaria de Humanidades, Educación, Ciencia y Tecnología

Año VI. Vol. VI. №10. Enero - Julio 2020

Hecho el depósito de ley: pp201602FA4721

ISSN-L: 2542-3029; ISSN: 2610-802X

Universidad Nacional Experimental Francisco de Miranda (UNEFM). Santa Ana de Coro. Venezuela

Kevin Santiago Lima Haig; Dayra Arelis Segovia Pacheco; César Andrés, Guerrero Velásteguí;

Leonardo G. Ballesteros - López

DOI 10.35381/cm.v6i10.121

\title{
Cultura Organizacional. Una visión desde el plan de comunicación publicitaria para la provincia de Tungurahua
}

\section{Organizational culture. A vision from the advertising communication plan for the province of Tungurahua}

\author{
Kevin Santiago Lima Haig \\ santylima23.Ih@outlook.com \\ Universidad Técnica de Ambato \\ Ecuador \\ https://orcid.org/0000-0002-6949-7962 \\ Dayra Arelis Segovia Pacheco \\ day-sego@hotmail.com \\ Universidad Técnica de Ambato \\ Ecuador \\ https://orcid.org/0000-0003-0452-0188
César Andrés Guerrero Velásteguí
day-sego@hotmail.com
ca.guerrero@uta.edu.ec
Universidad Técnica de Ambato
Ecuador \\ https://orcid.org/0000-0001-8482-7205 \\ Leonardo G. Ballesteros - López \\ leonardoballesteros@uta.edu.ec \\ Universidad Técnica de Ambato \\ Ecuador \\ https://orcid.org/0000-0003-1014-9872
}

Recibido: 28/06/2019

Aprobado: 16/07/2019

\footnotetext{
* El artículo forma parte de los resultados del proyecto de investigación: "INFLUENCIA DE LA PUBLICIDAD EN EL COMPORTAMIENTO DEL CONSUMIDOR DEL SECTOR COMERCIAL DE LA PROVINCIA DE TUNGURAHUA"; aprobado mediante resolución de Consejo Universitario: 2386-CU-P-2017 del 21 de noviembre de 2017, en la Universidad Técnica de Ambato; coordinado por el Ing. MBA. Leonardo Gabriel Ballesteros López.
} 
Kevin Santiago Lima Haig; Dayra Arelis Segovia Pacheco; César Andrés, Guerrero Velásteguí; Leonardo G. Ballesteros - López

\title{
RESUMEN
}

El presente estudio tiene como objetivo proponer un plan de comunicación publicitaria con énfasis en la cultura organizacional de la provincia de Tungurahua. El problema que se identifica es que las empresas del sector comercial de la provincia de Tungurahua, no poseen un plan de comunicación publicitaria para sus campañas, puesto que lo realizan en su mayoría empíricamente. Dentro de la metodología, esta investigación es de carácter descriptiva, con un nivel de investigación básico y un enfoque mixto; apoyándose en una revisión de la literatura con los aportes más destacados de las variables de estudio. Además, se aplicó una encuesta estructurada a una muestra de 231 empresas comerciales específicamente de línea blanca y electrodomésticos, validándose dicho instrumento con el estadístico de fiabilidad Alpha de Cronbach. En los resultados, constan las etapas del plan publicitario propuesto, y en conclusiones se destaca la importancia de la utilización del mismo en el ámbito empresarial al llegar a un segmento de mercado establecido.

Palabras claves: Publicidad; Cultura organizacional; Comunicación publicitaria; Empresas comerciales

\begin{abstract}
The objective of this study is to propose an advertising communication plan with emphasis on the organizational culture of the province of Tungurahua. The problem that is identified is that the companies of the commercial sector of the province of Tungurahua, do not have an advertising communication plan for their campaigns, since they do it mostly empirically. Within the methodology, this research is descriptive, with a basic level of research and a mixed approach; based on a review of the literature with the most outstanding contributions of the study variables. In addition, a structured survey was applied to a sample of 231 commercial companies, specifically white goods and household appliances, and this instrument was validated with the Cronbach Alpha reliability statistic. The results include the stages of the proposed advertising plan, and the conclusions highlight the importance of using it in the business field when reaching an established market segment.
\end{abstract}

Key words: Advertising; Organizational culture; Advertising communication; Commercial companies

\section{INTRODUCCIÓN}

Las empresas del sector comercial a nivel mundial aportan a la matriz productiva notablemente; puesto que generan mayores ingresos y fuentes de trabajo, gracias al manejo óptimo de la imagen de las empresas (Masaquiza, Ballesteros, \& González, 
2017); (Lascano-Cevallos, Castillo-Pérez, Mena-Méndez, \& Vayas-Ruiz, 2018). Por ello, los especialistas en marketing deben estar a la vanguardia de los nuevos medios digitales para utilizarlos y aplicarlos en el desarrollo de planes publicitarios.

La publicidad es una comunicación estructurada y un esfuerzo pagado, que contiene información no personal y persuasiva sobre productos, servicios o de una marca por anunciantes, que se difunden a través de diferentes medios masivos de comunicación (Arens, Weigold, \& Arens, 2008); (O’Guinn, Allen, \& Semenik, 2013), su importancia radica en que apoya la construcción en la imagen de la marca, informa y persuade con contenido específico, consolida otros esfuerzos de marketing, eleva la conciencia de marca, afirma la imagen de la marca y atrae segmentos específicos de clientes (Clow \& Baack, 2010); (Vallet et al., 2015), así se produce una constante innovación de la comunicación publicitaria con la finalidad de captar las exigencias del consumidor, (Maya, 2019), plantea que en ese caso se debe innovar con dirección a fin de evitar el caos.

Aunado a lo planteado, (Ortega Polanco, 2019), destaca que "si en los mensajes que transmiten las organizaciones se explota el uso de las emociones de manera correcta, se logrará la construcción de una imagen corporativa positiva, al estar influyendo directamente en la construcción de la percepción" (p. 151), de allí que las empresas deban trabajar la publicidad desde diversas ópticas para enfocar mejores resultados, en ese sentido, (Cevallos-Delgado, Limonta, \& Dueñas-Espinoza, 2017) concuerdan que los tipos de publicidad son: 1.- por audiencia seleccionada para el consumidor y para negocios; 2.- por área geográfica, siendo local, regional, nacional e internacional; 3.- Por medios, impresos, transmitidos, fuera del hogar, por correo directo e interactivo; 4.- por propósito, del producto, comercial, no comercial, de acción o de conciencia. Sin embargo, para (Ferrer \& Medina, 2014); (Cevallos-Delgado et al., 2017) agregan que se divide en publicidad tradicional o ATL (above the line) a través de revistas, periódicos y televisión; y publicidad en medios no convencionales o BTL (below the line), utilizándose otros tipos de soportes como: mailings, folletos, ferias $\mathrm{y}$ sponsors. 
Por otra parte, la cultura organizacional nacida en el siglo XIX, actualmente es un sector importante del mundo de los negocios y en el ámbito empresarial, pues las ganancias que obtienen las empresas no siempre se basan en una publicidad honesta (Bunge, 2011). Además, una de las funciones primordiales de la cultura comercial es ofrecer productos y servicios con valor agregado, garantizando utilidades para una empresa (Cuartas \& Pérez-Torre, 2017) ; (Huacallo-Valdez, 2018) ; (Duan, Ding, Hou, \& Zhang, 2018). Por lo tanto, es primordial en una sociedad altamente globalizada que está expuesta a la producción en serie de publicidad, como: radio, televisión, prensa, cine y medios sociales; para contribuir con las necesidades de los clientes. Por ésta razón, la cultura organizacional y la publicidad se relacionan para brindar un mensaje claro, realista, verdadero y conciso a los consumidores y clientes.

Una de las principales funciones de la comunicación publicitaria es la de informar, como lo menciona (Cevallos-García, 2016) ; (Sainz de Vicuña Ancín, 2018) ; (Singh \& Duhan, 2016), que permite compartir valiosa información y de ésta forma se utilizan medios de comunicación para transmitir el mensaje a la audiencia especifica (GarcíaLópez, 2017); (Ruíz -Hernández, Beverido-Sustaeta, Salas-García, \& Ortiz-León, 2018). Entonces, la comunicación publicitaria conlleva a la transmisión del mensaje eficientemente, a través de diferentes medios; razón por la que, es fundamental orientar un plan de comunicación publicitaria.

Cabe agregar que, los aportes de (Masaquiza et al., 2017) detallan que un plan publicitario posee las siguientes fases: análisis interno y externo, diagnostico situacional, directrices estratégicas, plan creativo, de medios, marketing digital, implementación, monitoreo y evaluación, y control. Además, para (Josemaría \& Bedoya, 2017) detallan los siguientes pasos para un plan de comunicación publicitaria: diagnóstico y definición de objetivos, publico objetivo, estrategias de comunicación, construcción del mensaje, alineamiento del mensaje, selección de medios, presupuesto, calendario y control de acciones. Sin embargo, estos autores se enfocan en la parte publicitaria, olvidándose la comunicación interna de una empresa. Por ello, es pertinente el desarrollo de un plan publicitario con un enfoque de cultura organizacional. 
Por otro lado, la comunicación organizacional involucra varias etapas: diagnóstico (del público, interna, externa y de la competencia), planificación (comité de comunicación, objetivos, estrategias, acciones, calendario, presupuesto, entrenamiento, educación, comunicación y despliegue de políticas), implementación de actividades y control (identifica fallas) (A. de Castro, 2017). Y engloba cuatro aspectos primordiales, como: 1.- misión, refiriéndose como la razón de existir y ser de una empresa, respondiendo cuatro preguntas: ¿quiénes somos?, ¿qué hacemos?, ¿para quién lo hacemos?, y ¿por qué lo hacemos?; 2.- visión: conlleva a una proyección del futuro de una empresa, a partir de la misión; definiéndose lo que la empresa desea alcanzar; 3.filosofía: permite orientar la empresa hacia el éxito de forma planeada, siendo ágil, dinámica y flexible; 4.- valores: son principios de una cultura de trabajo, para guiar al éxito de una empresa (Cevallos-García, 2016). A su vez, para (Kirkpatrick, 2001) una comunicación eficiente se basa en los siguientes componentes: credibilidad, contexto, contenido, claridad, continuidad y consistencia, canales de comunicación y capacidad del auditorio.

En ese sentido, el uso de los medios de comunicación en el Ecuador ha tenido un cambio drástico, puesto que de acuerdo al Instituto de Estadísticas y Censos (INEC, 2016) los teléfonos celulares poseen un $97,2 \%$ de utilización en las empresas, seguido del correo electrónico $95,3 \%$ y redes sociales con un $58,5 \%$. Asimismo, las empresas ecuatorianas destinan un $55 \%$ de sus recursos en publicidad display, $12 \%$ en publicidad móvil y $33 \%$ en publicidad a través de redes sociales (E. Castro, 2014). Por lo tanto, las empresas deben seleccionar adecuadamente los medios que utilizarán para sus campañas publicitarias con preferencia en las tendencias actuales.

El problema que se identifica es que las empresas del sector comercial de la provincia de Tungurahua, no poseen un plan de comunicación publicitaria para sus campañas, puesto que lo realizan en su mayoría empíricamente. Por esta razón, el objetivo de esta investigación es proponer un plan de comunicación publicitaria con énfasis en la cultura organizacional de la provincia de Tungurahua. 


\section{METODOLOGÍA}

Al ser un estudio descriptivo, se busca identificar características específicas de la sociedad sin cambiar el estado natural de dicha sociedad; y el nivel de investigación que se aplica es el básico, ya que tiene como finalidad la obtención y recopilación de información para ir construyendo una base de conocimiento que se va agregando a la información previa existente. Además, este estudio se fundamenta en un enfoque de investigación mixto. Para ello, se realizó una revisión de la literatura con los aportes más destacados de la cultura organizacional y la comunicación publicitaria.

La población de estudio son 3,186 empresas dedicadas exclusivamente al sector comercial, de las cuales 577 son empresas comerciales de línea blanca y electrodomésticos de la provincia de Tungurahua, con una muestra de 231 empresas a quienes se aplicó una encuesta estructurada. También, se validó el cuestionario con el estadístico de fiabilidad Alpha de Cronbach, obteniéndose un resultado de 0.88 , evidenciándose un cuestionario fiable.

\section{RESULTADOS}

Después de las consideraciones bibliográficas anteriores, se plantean las siguientes etapas para el desarrollo de un plan de comunicación publicitaria con énfasis en la cultura organizacional para empresas comerciales de línea blanca y electrodomésticos de la provincia de Tungurahua: diagnóstico situacional, análisis interno, aspectos de cultura organizacional, definición de objetivos y estrategias, público objetivo, construcción y alineamiento del mensaje, selección de canales de comunicación, presupuesto, calendario, ejecución, control y evaluación.

\section{Diagnóstico Situacional}

El $50 \%$ de las empresas encuestadas, señala estar de acuerdo en que la cultura organizacional es importante para las empresas y un 30\% está totalmente de acuerdo. Además, especifican que es importante la cultura organizacional para las empresas por el trabajo en equipo 35\%, por la toma de decisiones $30 \%$, por la innovación un 
$15 \%$, por los principios un $10 \%$ y por los valores y el liderazgo $5 \%$ respectivamente (ver gráfico 1). Por ello, las empresas del sector comercial, específicamente de línea blanca y electrodomésticos deben tener en cuenta varios elementos de la cultura organizacional, como: la mejora continua 55\% y la comunicación 35\%.

Gráfico 1. Importancia de la cultura organizacional

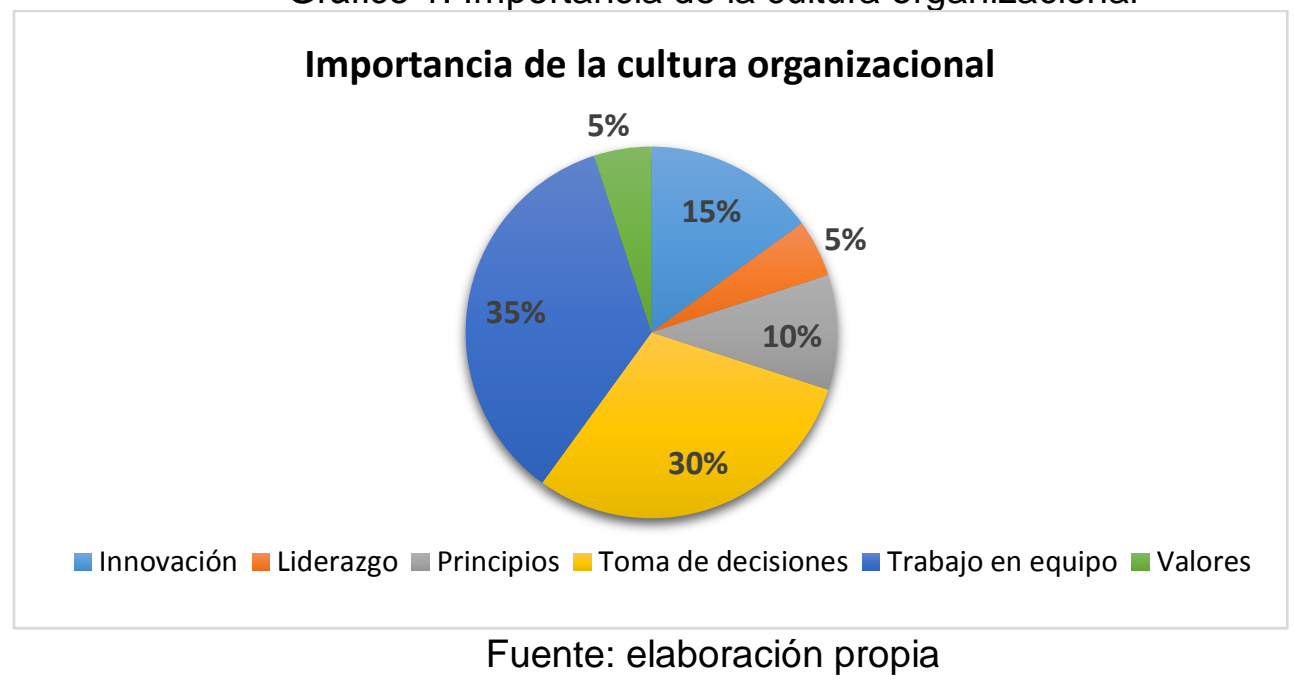

El $50 \%$ de los encuestados está de acuerdo en que la cultura organización influye en la comunicación publicitaria. En donde, la comunicación publicitaria 50\% que corresponde a 116 empresas y la percepción de los productos o servicios $40 \%$ que equivale a 93 empresas, seguido de los valores y de los comentarios de los empleados con un $5 \%$ que equivale a 11 empresas, señalan que son aspectos fundamentales para que se promueva la cultura organizacional de las empresas del sector comercial, específicamente de línea blanca y electrodomésticos a los clientes y consumidores (ver gráfico 2).

Gráfico 2. Cultura organizacional en el sector comercial

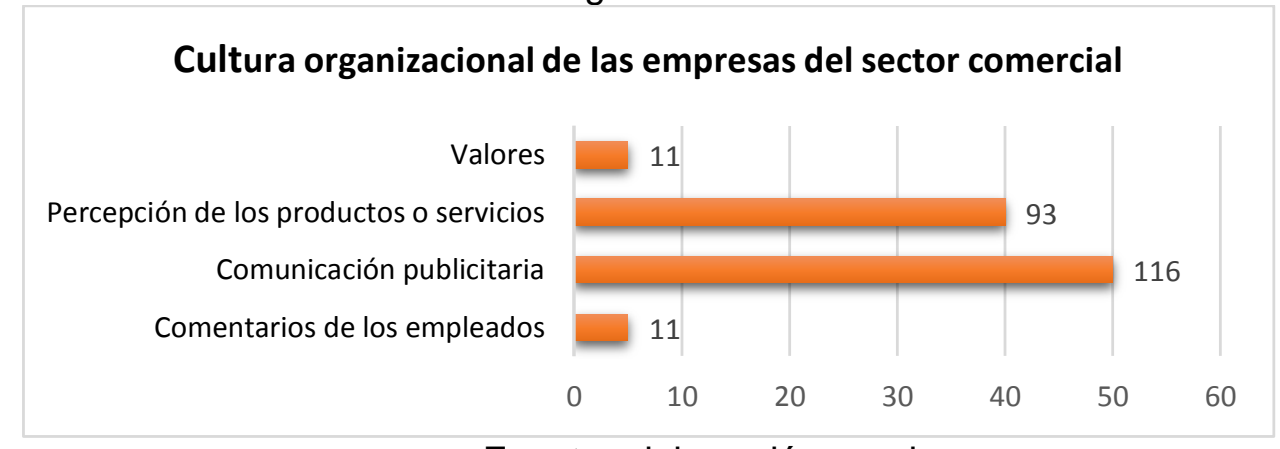

Fuente: elaboración propia 
Las empresas objeto de estudio consideran buenas en un 50\% las formas tradicionales de publicidad, como: volantes, vallas publicitarias, radio, prensa, televisión, entre otros. Publicitando con más frecuencia a su empresa con un 55\%; mientras que un $45 \%$ publicita específicamente los productos que oferta al mercado. También, como tipo de publicidad para promocionarse las empresas utilizan en un $45 \%$ las redes sociales equivalente a 105 empresas, un $25 \%$ los volantes correspondiente a 58 empresas, un 15\% las vallas publicitarias equivalente a 35 empresas y un $5 \%$ correspondiente a 11 empresas utiliza televisión, radio y prensa respectivamente (ver gráfico 3). Sin embargo, estas empresas planifican su inversión publicitaria de acuerdo a las ventas $40 \%$ y de acuerdo a la demanda de sus productos en un $35 \%$.

Gráfico 3. Tipo de publicidad

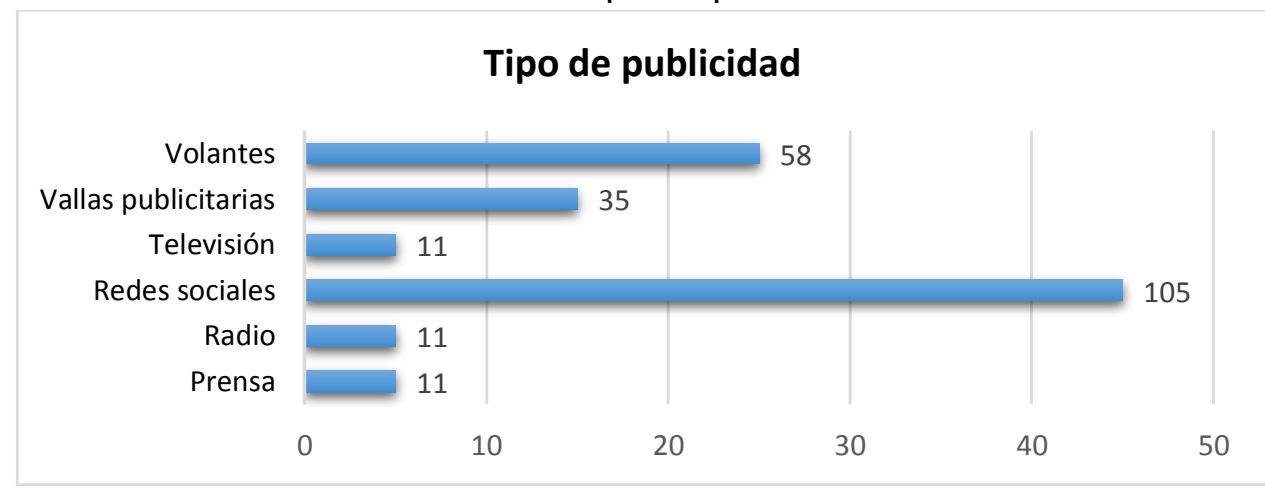

Fuente: elaboración propia

Los clientes que con más frecuencia adquieren los productos y servicios son consumidores finales con un $60 \%$ y pequeños comerciantes con un $30 \%$. Y lo hacen por los descuentos y promociones con un $40 \%$ y por la calidad con un $30 \%$, seguido de las facilidades de pago con un $20 \%$.

Por otro lado, las empresas poseen ventas medianamente redituables con un 55\%. Por ello, deben enfocarse en los planes publicitarios para dar a conocer y promocionar sus marcas. En ese sentido, el $85 \%$ de las empresas utilizaría los planes de comunicación publicitaria en su empresa y el $60 \%$ está de acuerdo en utilizar un plan 
de comunicación con énfasis en la cultura comercial específicamente para el sector comercial de línea blanca y electrodomésticos.

\section{Análisis interno y externo}

Esta etapa conlleva el análisis interno (FODA) y externo (PESTEL) de las empresas, para lo cual se detalla a continuación:

\section{Cuadro 1. Matriz FODA de la empresa comercial Baratodo}

\section{FACTORES INTERNOS}

\section{FORTALEZAS}

F1: Capacitación de los miembros de la empresa.

F2: Productos de calidad, costos accesibles, variedad.

F3: Óptimo servicio al cliente y asesoría en los locales comerciales.

F4: Utiliza medios digitales para publicidad y el merchandising (exhibición de mercadería) en los locales.

F5: Planificación de inversión publicitaria de acuerdo a la demanda de los productos

\section{OPORTUNIDADES}

01: Gran demanda de los productos de línea blanca y electrodomésticos.

O2: Marca representativa del sector

O3: Alianzas estratégicas con proveedores mayoristas nacionales.

O4: Apoyo gubernamental a las empresas

\section{DEBILIDADES}

D1: Falta de trabajo en equipo, unión y toma de decisiones

D2: Competitividad en productos de otras empresas.

D3: Insuficiente asesoría de las características de los productos en medios digitales.

D4: No evalúa los medios publicitarios, utiliza sólo redes sociales.

D5: No utiliza planes publicitarios.

Fuente: elaboración propia

Por tanto, la empresa comercial Baratodo posee productos de alta calidad, precio accesible y gran variedad de marcas para que el consumidor pueda escoger a su gusto y de acuerdo a las características que necesite. Para ello, la empresa brinda un servicio al cliente de calidad y asesoría en los locales comerciales y los publicita a través de medios digitales y el merchandising (exhibición de mercadería) en los locales. 


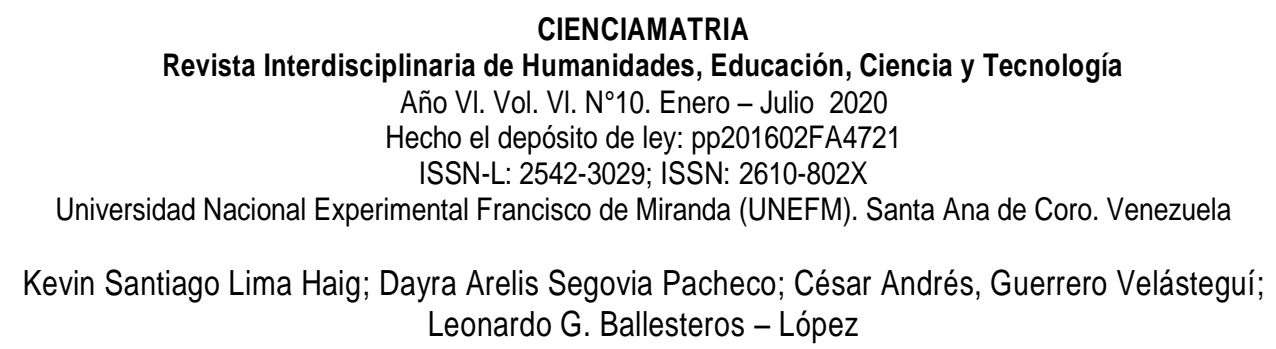

Sin embargo, se debe ampliar los medios de publicidad y no sólo enfocarse en redes sociales, puede optar por vallas publicitarias, volanteo, radio y televisión, como publicidad tradicional, pero se puede utilizar otros tipos de marketing como: códigos $\mathrm{QR}$, realidad aumentada y publicidad en lugares masivos como cines y autobuses. Por otro lado, la gran demanda de los productos de línea blanca y electrodomésticos es una ventaja y gran oportunidad en un mercado muy competitivo. Por ésta razón, la empresa debe realizar conversatorios para concretar alianzas estratégicas con los proveedores, con la finalidad de conseguir precios más bajos y obtener un margen de ganancia superior.

\section{Cuadro 2. Análisis PEST de la empresa comercial Baratodo \\ POLITICO \\ ECONÓMICO}

- Políticas comerciales para fortalecer los mercados internos.

- Plan Nacional de Desarrollo promueve el aparato productivo y la producción nacional, y los emprendimientos.

- Protección de la economía ecuatoriana.

SOCIOCULTURAL

- El $61 \%$ de las compras son realizadas en los locales.

- El $25 \%$ de las compras se realizan por promociones y descuentos.

- Los grupos que más compran por internet son entre 25-45 años con el $59,4 \%$.

Fuente: elaboración propia a partir de (Asamblea Nacional, 2008); (BCE, 2018); (Tapia \& Castillo,

- Crecimiento del PIB en un $38 \%$

- Aporte del sector comercial en un $44,34 \%$ del PIB

- En 2018 el sector comercial alcanzó 151,003 millones de dólares.

- Generación de empleo de calidad en el sector comercial 16\% para el 2018.

\section{TECNOLÓGICO}

- Utilización de P.S.P (Point of Sale) para compras con tarjetas de débito y crédito con 584,2 millones de dólares en transacciones.

- El $47,2 \%$ de empresas invirtieron en Tecnologías de la Información y Comunicación en el 2016.
2018)

En el cuadro 2 se evidencia, que los aspectos políticos son fundamentales para las empresas nuevas, y que desean posicionarse en el mercado. Así, las políticas comerciales, de acuerdo a la (República del Ecuador Comité de Comercio Exterior, 2014) en el Art. 304 estipula que "la política comercial tendrá el objetivo de desarrollar, fortalecer, dinamizar los mercados internos a partir del objetivo estratégico establecido en el Plan Nacional de Desarrollo, para fortalecer el aparato 
productivo y la producción nacional" (p.1). Y en referencia a la (República del Ecuador Comité de Comercio Exterior, 2015) dentro de las políticas adoptadas por el gobierno para la protección de la economía ecuatoriana figura el tema de las salvaguardias que según el comité de comercio exterior, recomienda el ámbito y niveles de la sobretasa arancelaria necesaria para salvaguardar el equilibrio de la balanza de pagos.

Además gracias al crecimiento evidente del PIB (Producto Interno Bruto), existe un aporte considerable del sector comercial dentro de éste y de la matriz productiva. También, se destaca que el perfil consumidor se enfoca en promociones y descuentos, así como, la compra directamente en los locales y con el pago a través de tarjeta de crédito o débito. Por lo cual se debe establecer estrategias específicas para atraer consumidores potenciales sean al local y por medios convencionales y no convencionales.

\section{Aspectos de cultura organizacional}

Misión: Baratodo una marca de Grupo Unicomer, que lidera sus negocios actuando de manera justa, transparente y honesta. Este es un compromiso que asumimos con nuestros empleados, clientes, proveedores y con la sociedad.

Visión: Baratodo busca liderar el mercado ecuatoriano en la comercialización con rentabilidad de bienes y servicios, con responsabilidad social.

Valores: Honestidad, transparencia, lealtad, empatía, responsabilidad, servicio.

Recursos humanos: la empresa comercial debe realizar periódicamente ciertas actividades para que los colaboradores internos, estén a gusto dentro de la misma. Así, todas las acciones empresariales estarán enfocadas a la comunicación asertiva, trabajo en equipo, servicio al cliente, manejo de conflictos, respeto a las diferencias, creatividad innovadora, toma de decisiones y liderazgo, todos dentro de la cultura organizacional.

- Reuniones informativas: en cada departamento para establecer los objetivos de cada uno de ellos y entre todos aportar en soluciones viables. De ésta forma se 
Kevin Santiago Lima Haig; Dayra Arelis Segovia Pacheco; César Andrés, Guerrero Velásteguí; Leonardo G. Ballesteros - López

consolida el compromiso interno y el trabajo en equipo, a través de una comunicación asertiva. Similarmente, tomar en cuenta las sugerencias del personal; así como, las soluciones que ellos presenten; consolidándose y promoviéndose la creatividad e innovación del personal a través de sus criterios, aportes y sugerencias.

- Talleres de capacitación: en todas las áreas, específicamente en atención al cliente, servicio al cliente, relaciones públicas y técnicas de ventas, con la finalidad de capacitar constantemente al personal, lográndose una mejora continua.

- Actividades de compañerismo: con los cuales los colaboradores se comprometan con la misión y visión de la empresa. De ésta forma, se consolidará el sentimiento de pertenencia empresarial y el trabajo en equipo.

- Manejo de conflictos: los jefes departamentales conjuntamente con el departamento de recursos humanos aplicarán varias técnicas de manejo de conflictos: 1.- hablar con las personas involucradas; 2.-escucharlas con atención; 3.-identificar los puntos de acuerdo y desacuerdo; 4.-priorizar las áreas de conflicto; 5.- desarrollar un plan de trabajo en los desacuerdos; 6.- celebrar el éxito.

- Promover la tolerancia y el respeto en los criterios y formas de pensar de cada uno de los colaboradores internos. De esta forma, existe no solo trabajo en equipo sino diversidad de pensamientos, los cuales pueden aportar cada uno a su manera a la consecución de los objetivos empresariales.

\section{Definición de objetivos y estrategias}

Se establece los siguientes objetivos y estrategias:

Cuadro 3. Definición de objetivos y estrategias

\section{OBJETIVOS}

- Incrementar las ventas en un $3 \%$ de la empresa para el mes de Mayo 2019.

- Aumentar la fidelidad de los clientes de un 30 por 100 a un 45 por 100 para el mes de Mayo 2019.
ESTRATEGIA

- Elaborar un plan de
comunicación publicitaria
enfocado en la cultura
organizacional.




\section{Público objetivo}

Las variables para definir el público objetivo son: edad, nacionalidad, ingresos, nivel socioeconómico, beneficios buscados, modalidad de compra, estilos de vida.

- Edad: personas entre 25-45 años.

- Nacionalidad: Ecuatoriana - Ambateños

- Ingresos: de \$394 en adelante

- Nivel socioeconómico: medio y medio alto

- Beneficios buscados: calidad en los productos, variedad, precios accesibles.

- Modalidad de compra: compra en los locales comerciales, pago en efectivo o tarjetas de crédito o débito, o a plazos.

- Estilo de vida: familiar

\section{Construcción y alineamiento del mensaje}

Slogan: ¡Mamita llévatelo todo!

Mensaje: ¡Aprovecha los descuentos por el mes de Mamá y renueva tu hogar con estas promociones!

Fechas de la campaña: mes de Mayo de 2019.

Ilustración 1. Publicidad de la empresa comercial Baratodo

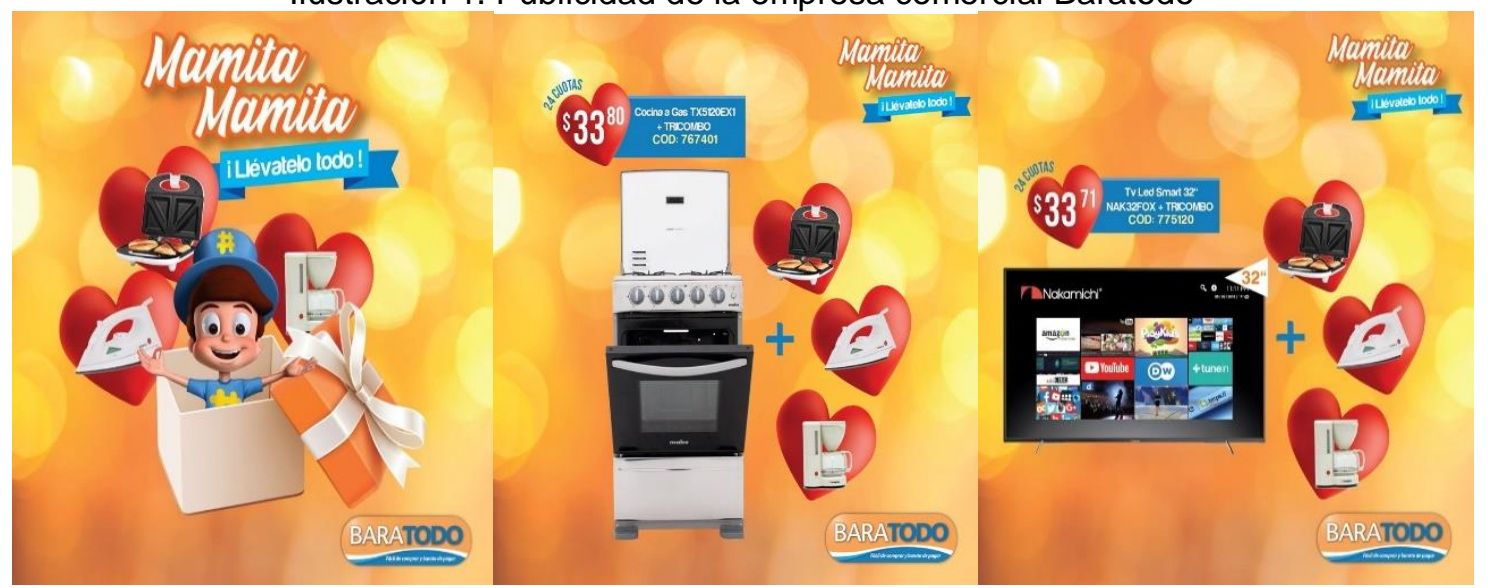

Fuente: (Baratodo, 2019)

\section{Promoción de ventas:}

- Concurso y sorteo: La empresa realiza actividades que proporcionan a los clientes y consumidores la oportunidad de ganar un tri-combo, en donde quienes acierten en el laberinto, se les sorteará el premio. 


\section{CIENCIAMATRIA}

Revista Interdisciplinaria de Humanidades, Educación, Ciencia y Tecnología Año VI. Vol. VI. №10. Enero - Julio 2020

Hecho el depósito de ley: pp201602FA4721

ISSN-L: 2542-3029; ISSN: 2610-802X

Universidad Nacional Experimental Francisco de Miranda (UNEFM). Santa Ana de Coro. Venezuela

Kevin Santiago Lima Haig; Dayra Arelis Segovia Pacheco; César Andrés, Guerrero Velásteguí; Leonardo G. Ballesteros - López

Mensaje: Selecciona el camino correcto y cuéntanos en los comentarios cuál te lleva al tri-combo gratis.

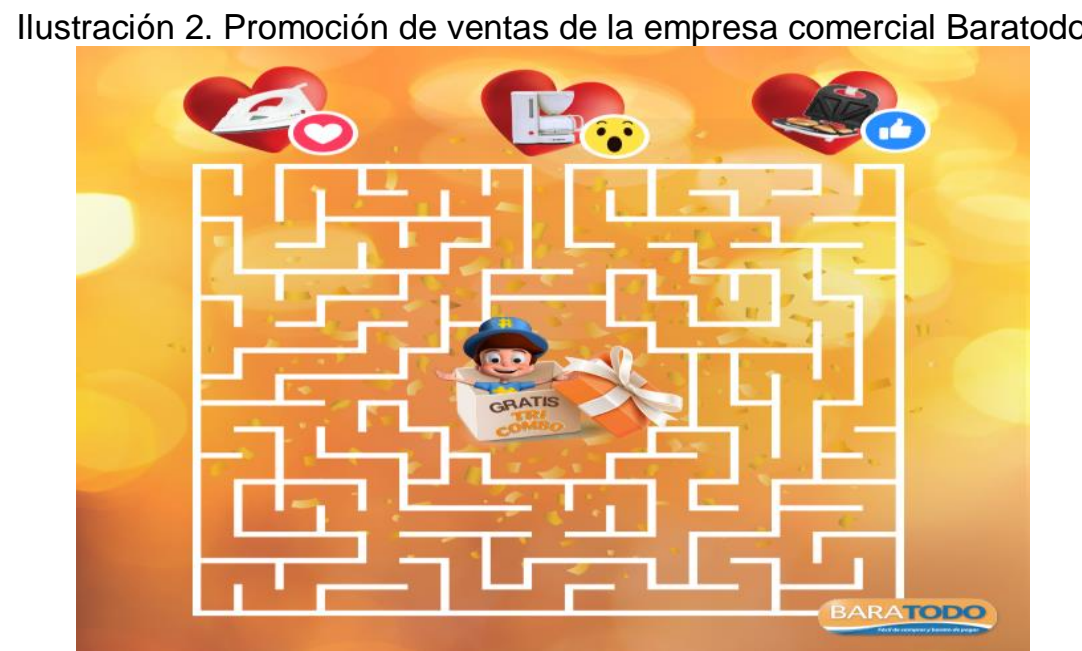

Fuente: (Baratodo, 2019)

- Mailing: la empresa comercial enviará esta información publicitaria a los buzones de correo de sus clientes existentes, para lo cual utilizará la bases de datos interna.

- Telemarketing. La empresa ofrecerá sus servicios por teléfono; donde el asesor comercial llamará al cliente con el fin de generar una respuesta de venta.

\section{Selección de canales de comunicación}

De acuerdo con la encuesta aplicada a las empresas, un alto porcentaje se evidenció el uso de las redes sociales como principal medio de comunicación. Es así, que se determinó que el medio por excelencia para el mensaje publicitario son las redes sociales, específicamente Facebook, aunque se precisa de otros medios la complementen: radio, volanteo, correo electrónico y venta por teléfono. 
Revista Interdisciplinaria de Humanidades, Educación, Ciencia y Tecnología Año VI. Vol. VI. №10. Enero - Julio 2020

Hecho el depósito de ley: pp201602FA4721

ISSN-L: 2542-3029; ISSN: 2610-802X

Universidad Nacional Experimental Francisco de Miranda (UNEFM). Santa Ana de Coro. Venezuela

Kevin Santiago Lima Haig; Dayra Arelis Segovia Pacheco; César Andrés, Guerrero Velásteguí; Leonardo G. Ballesteros - López

\section{Presupuesto}

En éste plan se utilizará el siguiente presupuesto:

Cuadro 4. Presupuesto de medios publicitarios

\begin{tabular}{|c|c|c|c|c|c|}
\hline \multirow{2}{*}{$\begin{array}{c}\text { Medios } \\
\text { publicitarios }\end{array}$} & \multirow{2}{*}{ Emisora } & \multirow{2}{*}{ Programa } & \multirow{2}{*}{$\begin{array}{c}\text { Fecha } \\
\text { Mayo } 2019\end{array}$} & \multirow{2}{*}{\multicolumn{2}{|c|}{ Valor }} \\
\hline & & & & & \\
\hline \multirow[b]{2}{*}{ Radio } & $\begin{array}{l}\text { Radio Bandida } \\
89.7 \\
\text { 몽 }\end{array}$ & $\begin{array}{c}{ }^{*} \text { El garrotazo } \\
(4-6 a m) \\
{ }^{*} \text { Noches de } \\
\text { Clímax } \\
(18: 00-22: 00 \text { pm })\end{array}$ & $\begin{array}{c}\text { *10 spots diarios } \\
\text { de Lunes a } \\
\text { Viernes } \\
\text { *5 spots diarios } \\
\text { Bonificación } \\
\text { Sábado }\end{array}$ & $\$$ & 650.00 \\
\hline & $\begin{array}{c}\text { Radio Canela } \\
106.5 \\
\text { Cnela }\end{array}$ & $\begin{array}{c}{ }^{*} \text { El canelazo } \\
\text { mañanero } \\
(05: 00-07: 00 \mathrm{am}) \\
{ }^{*} \text { Habitantes de la } \\
\text { noche } \\
(19: 00-20: 00 \mathrm{pm})\end{array}$ & $\begin{array}{c}\text { *15 spots diarios } \\
\text { de Lunes a } \\
\text { Viernes } \\
\text { *4 spots diarios } \\
\text { diarias } \\
\text { Bonificación } \\
\text { Sábado }\end{array}$ & $\$$ & 750.00 \\
\hline Mailing & & & $\begin{array}{c}{ }^{*} \text { Tres } \\
\text { publicaciones } \\
\text { semanales }\end{array}$ & $\$$ & 50.00 \\
\hline $\begin{array}{l}\text { Redes } \\
\text { sociales }\end{array}$ & & & $\begin{array}{l}\text { *Una publicación } \\
\text { diaria }\end{array}$ & $\$$ & 120.00 \\
\hline Telemarketing & $\begin{array}{l}\text { Teléfono } \\
\text { empresarial }\end{array}$ & & $\begin{array}{c}\text { *Una publicación } \\
\text { diaria }\end{array}$ & $\$$ & 80.00 \\
\hline \multicolumn{4}{|c|}{ Total presupuesto } & $\$$ & 1650.00 \\
\hline
\end{tabular}

Fuente: elaboración propia

\section{Calendario}

El plan publicitario tiene el siguiente cronograma:

Cuadro 5. Cronograma del plan publicitario

\begin{tabular}{|c|c|c|c|c|c|c|c|c|c|c|c|}
\hline \multirow{2}{*}{ Tarea } & \multirow{2}{*}{ Fechas } & \multicolumn{10}{|c|}{ Semanas } \\
\hline & & 1 & 2 & 3 & 4 & 5 & 6 & 7 & 8 & 9 & 10 \\
\hline Diagnóstico Situacional & \multirow{3}{*}{$\begin{array}{l}9 \text { de abril }-23 \text { de } \\
\text { abril de } 2019\end{array}$} & & & & & & & & & & \\
\hline Análisis interno y externo & & & & & & & & & & & \\
\hline $\begin{array}{l}\text { Aspectos de cultura } \\
\text { organizacional }\end{array}$ & & & & & & & & & & & \\
\hline $\begin{array}{l}\text { Definición de objetivos y } \\
\text { estrategias }\end{array}$ & \multirow{2}{*}{$\begin{array}{l}16 \text { de abril }-30 \text { de } \\
\text { abril de } 2019\end{array}$} & & & & & & & & & & \\
\hline Público Objetivo & & & & & & & & & & & \\
\hline
\end{tabular}




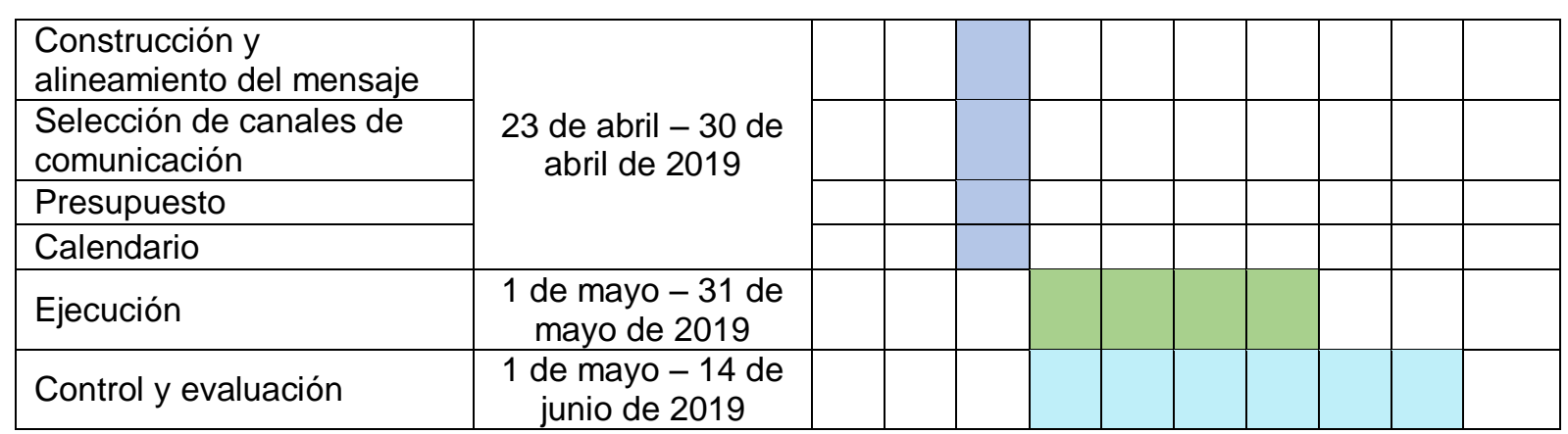

Fuente: elaboración propia

\section{Ejecución}

Éste plan publicitario tiene fechas de ejecución desde 1 de mayo - 31 de mayo de 2019, lo que equivale a todo un mes, 31 días, puesto que este mes es festivo por el día de la madre. Sin embargo, para planificar el plan publicitario se añaden tres semanas antes y para controlar y monitorear dicho plan se añaden tres semanas después.

Razón por la cual, el desarrollo y planificación, la ejecución, y el control y monitoreo conlleva a un total de 10 semanas exactas.

\section{Control y evaluación}

El control será durante todo el mes de ejecución y su evaluación durante dos semanas posteriores. Para ello, se utilizará fórmulas específicas para cada uno de los medios publicitarios: radio, mailing, redes sociales y telemarketing. 
Kevin Santiago Lima Haig; Dayra Arelis Segovia Pacheco; César Andrés, Guerrero Velásteguí; Leonardo G. Ballesteros - López

Cuadro 5. Correlación entre la importancia de la cultura organizacional y sus aspectos en la comunicación publicitaria

\begin{tabular}{|l|r|r|r|r|r|}
\hline & $\begin{array}{c}\text { Totalmente de } \\
\text { acuerdo }\end{array}$ & De acuerdo & $\begin{array}{c}\text { Ni de acuerdo ni } \\
\text { en desacuerdo }\end{array}$ & Total & $\%$ \\
\hline Innovación & 0 & 33 & 0 & 33 & $14 \%$ \\
Liderazgo & 0 & 11 & 0 & 11 & $5 \%$ \\
Toma de decisiones & 0 & 44 & 22 & 66 & $29 \%$ \\
Trabajo en equipo & 65 & 0 & 22 & 87 & $38 \%$ \\
Valores & 12 & 0 & 0 & 12 & $5 \%$ \\
Principios & 0 & 22 & 0 & 22 & $10 \%$ \\
Total & 77 & 110 & 44 & 231 & $100 \%$ \\
\hline
\end{tabular}

Fuente: herramienta SPSS

Se presenta la prueba de chi-cuadrado que se encarga de medir la influencia de variables cualitativas y el efecto de correlación e incidencia que posee una variable sobre la otra. Relacionándose las preguntas: 4. ¿Considera que la cultura organizacional influye en la comunicación publicitaria?, y 2. ¿Por qué es importante la cultura organizacional para las empresas? A partir de ésta relación se obtuvieron los siguientes resultados.

Cuadro 6. Correlación Pruebas de chi-cuadrado (empresas comerciales de línea blanca y electrodomésticos)

\begin{tabular}{|l|r|r|r|}
\hline & \multicolumn{1}{|c|}{ Valor } & \multicolumn{1}{|c|}{ gl } & $\begin{array}{c}\text { Sig. asintótica } \\
\text { (bilateral) }\end{array}$ \\
\hline Chi-cuadrado de Pearson & $218,597^{\mathrm{a}}$ & 10 &, 000 \\
Razón de verosimilitudes & 295,925 & 10 &, 000 \\
Asociación lineal por lineal & 12,702 & 1 &, 000 \\
N de casos válidos & 231 & & \\
\hline
\end{tabular}

Fuente: herramienta SPSS

Como muestra el cuadro 6, se obtuvo un chi-cuadrado de 218,597 a 10 grados de libertad, bajo este escenario se puede determinar que el chi-cuadrado calculado $(218,597)$ es mayor al chi-cuadrado tabulado 192,343. Por lo que se acepta la hipótesis alternativa y se rechaza la nula (ver figura 1), asumiéndose que las variables de estudio: la cultura organizacional y la comunicación publicitaria no son independientes y sí tienen correlación entre ellas; evidenciándose la importancia de ésta investigación al mostrar que las variables de estudio se interrelacionan entre sí. 


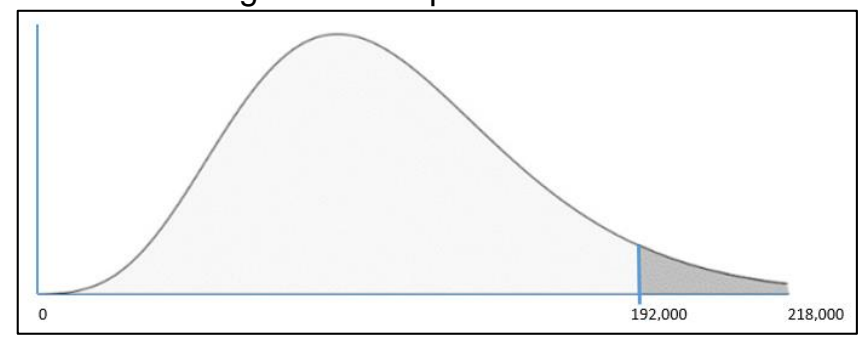

Fuente: herramienta SPSS

\section{CONCLUSIONES}

La comunicación publicitaria apoya a la difusión y compartición de información a través de un mensaje claro y conciso. Además, se utilizan medios de comunicación sean convencionales o no convencionales, los cuales permitan llegar a un segmento de mercado establecido.

La cultura organizacional, está inmersa dentro de todos los procesos administrativos de una empresa; así como, al aplicar en la publicidad, puesto que éste refleja la esencia de la empresa, refleja su organización, planificación, trabajo en equipo, liderazgo y comunicación asertiva. Por ello, al trabajar en aspectos organizacionales internos, implícitamente, se trabaja en lo que la empresa proyecta al mercado y a los clientes.

En ese sentido, esta investigación propone un plan de comunicación publicitaria con énfasis en la cultura organizacional de la provincia de Tungurahua, el cual posee las once etapas: 1.-diagnóstico situacional; 2.-análisis interno; 3.-aspectos de cultura organizacional; 4.-definición de objetivos y estrategias; 5.-público objetivo; 6.construcción y alineamiento del mensaje; 7.-selección de canales de comunicación; 8.- presupuesto; 9.-calendario; 10.-ejecución; 11.-control y evaluación. 
Kevin Santiago Lima Haig; Dayra Arelis Segovia Pacheco; César Andrés, Guerrero Velásteguí; Leonardo G. Ballesteros - López

\section{REFERENCIAS CONSULTADAS}

1. Arens, W., Weigold, M., \& Arens, C. (2008). Publicidad (Undécima). México, D.F: McGraw-Hill Interamericana.

2. Asamblea Nacional. (2008). Constitución de la República del Ecuador. Recuperado de https://www.oas.org/juridico/pdfs/mesicic4_ecu_const.pdf

3. Baratodo. (2019). Baratodo - Publicaciones. Recuperado 26 de mayo de 2019, de https://www.facebook.com/pg/baratodoecuador/posts/?ref=page_internal

4. BCE. (2018). Producto Interno Bruto. Recuperado 14 de junio de 2017, de https://www.bce.fin.ec/index.php/component/k2/item/310-producto-internobruto

5. Bunge, M. (2011). 100 ideas. Buenos Aires, Argentina: Penguin Random House Grupo Editorial Argentina.

6. Castro, A. de. (2017). Manual práctico de Comunicación Organizacional. Barranquilla, Colombia: Editorial Verbum.

7. Castro, E. (2014). Ecuador informe anual de inversión publicitaria en Internet 2013. IAB.

8. Cevallos-Delgado, M. D., Limonta, R. J., \& Dueñas-Espinoza, F. (2017). Análisis de los ser vicios pub licitar ios deman dados por las em presas públicas y privadas de la ciudad de Portoviejo. Revista San Gregorio, (19), 88-100.

9. Cevallos-García, I. (2016). La comunicación publicitaria en la cultura académica universitaria bajo los parámetros de la educación actual. Revista Electrónica Formación y Calidad Educativa (REFCalE), 10.

10. Clow, K., \& Baack, D. (2010). Publicidad, promoción y comunicación integral en marketing (Cuarta). México: Pearson Prentice Hall.

11. Cuartas, S. A., \& Pérez-Torre, M. É. (2017). El pediatra como nexo bioético entre la publicidad infantil y el entorno familiar. Revista Cubana de Pediatría., 89(4), 12.

12. Duan, T., Ding, R., Hou, W., \& Zhang, J. Z. (2018). The burden of attention: CEO publicity and tax avoidance. Journal of Business Research, 87, 90-101. https://doi.org/10.1016/j.jbusres.2018.02.010

13. Ferrer, I., \& Medina, P. (2014). Gestión empresarial de la Agencia de Publicidad. Madrid, España: Diaz de Santos. 
Kevin Santiago Lima Haig; Dayra Arelis Segovia Pacheco; César Andrés, Guerrero Velásteguí; Leonardo G. Ballesteros - López

14. García-López, J. (2017). Publicidad, necesidades y decrecimiento. Hacia una comunicación publicitaria menos comercial. Chasqui Revista Latinoamericana de Comunicación, (134), 16.

15. Huacallo-Valdez, L. A. (2018). Plan de negocio para la implementación de un nuevo medio de publicidad digital en vehículos de transporte urbano en la ciudad de Arequipa. Universidad Nacional de San Agustín, Arequipa, Perú.

16. INEC. (2016). Tecnologías de la Información y Comunicaciones (TIC's) 2016. Recuperado de http://www.ecuadorencifras.gob.ec/documentos/webinec/Estadisticas_Sociales/TIC/2016/170125.Presentacion_Tics_2016.pdf

17. Josemaría, J. A., \& Bedoya, A. M. de. (2017). Plan de comunicación on y off en la práctica. Madrid, España: ESIC Editorial.

18. Kirkpatrick, D. L. (2001). Claves para una comunicación eficaz. Barcelona, España: Gestión 2000.

19. Lascano-Cevallos, A. D., Castillo-Pérez, E. I., Mena-Méndez, D., \& Vayas-Ruiz, E. C. (2018). La Fiesta de la fruta y las flores en el escenario local de Ambato: análisis de la comunicación publicitaria (2012-2017). Revista de Estudios Urbanos y Ciencias Sociales, 8(1), 61-77.

20. Masaquiza, C., Ballesteros, L., \& González, L. (2017). Análisis de los stakeholders como insumo de entrada en el diseño de un plan publicitario. Universidad, Ciencia y Tecnología, 1, 60-67.

21. Maya, K. (2019). Innovación para el desarrollo económico del Gremio Artesanal. Revista Arbitrada Interdisciplinaria Koinonía, 4(7), 88-102. doi:http://dx.doi.org/10.35381/r.k.v4i7.195

22. O'Guinn, T., Allen, C., \& Semenik, R. (2013). Publicidad y Promoción Integral de marca (6ta ed.). México, D.F: Cengage Learning Editores.

23. Ortega Polanco, V. (2019). Gestión de la imagen corporativa de organizaciones universitarias desde el enfoque del marketing emocional. CIENCIAMATRIA, 3(5), 150-171. Recuperado a partir de http://www.cienciamatriarevista.org.ve/index.php/cm/article/view/19

24. República del Ecuador Comité de Comercio Exterior. Resolución № 050-2014 (2014).

25. República del Ecuador Comité de Comercio Exterior. Resolución № 011-2015 (2015). 
26. Ruíz -Hernández, A. R., Beverido-Sustaeta, P., Salas-García, B., \& Ortiz-León, M. C. (2018). Publicidad y consumo de tabaco en mujeres de la Ciudad de México. Revista Médica de la Universidad Veracruzana, 18(1).

27. Sainz de Vicuña Ancín, J. M. (2018). El plan de marketing digital en la práctica. ESIC Editorial.

28. Singh, A., \& Duhan, P. (Eds.). (2016). Managing Public Relations and Brand Image through Social Media: https://doi.org/10.4018/978-1-5225-0332-3

29. Tapia, E., \& Castillo, A. (2018). Locales deben aceptar todas las tarjetas. Recuperado 26 de mayo de 2019, de El Comercio website: https://www.elcomercio.com/actualidad/locales-tarjetas-credito-debitocomercio.html

30. Vallet, T., Vallet, A., Vallet, I., Casanova, E., Del Corte, V., Estrada, M., \& Monte, P. (2015). Principios de marketing estratégico. Castellón de la Plana: Universitat Jaume I.

\section{REFERENCES CONSULTED}

1. Arens, W., Weigold, M., \& Arens, C. (2008). Advertising (Eleventh). Mexico, D.F: McGraw-Hill Interamerican.

2. National Assembly. (2008). Constitution of the Republic of Ecuador. Recovered from https://www.oas.org/juridico/pdfs/mesicic4_ecu_const.pdf

3. Cheap (2019). Cheap - Publications. Retrieved May 26, 2019, from https://www.facebook.com/pg/baratodoecuador/posts/?ref=page_internal

4. ECB. (2018). Gross domestic product. Retrieved June 14, 2017, from https://www.bce.fin.ec/index.php/component/k2/item/310-producto-internobruto

5. Bunge, M. (2011). 100 ideas Buenos Aires, Argentina: Penguin Random House Editorial Group Argentina.

6. Castro, A. of. (2017). Practical Manual of Organizational Communication. Barranquilla, Colombia: Editorial Verbum.

7. Castro, E. (2014). Ecuador annual report on advertising investment on the Internet 2013. IAB. 
8. Cevallos-Delgado, M. D., Limonta, R. J., \& Dueñas-Espinoza, F. (2017). Analysis of the public services tendered by the public and private companies of the city of Portoviejo. San Gregorio Magazine, (19), 88-100.

9. Cevallos-García, I. (2016). Advertising communication in university academic culture under the parameters of current education. Electronic Magazine Training and Educational Quality (REFCalE).

10. Clow, K., \& Baack, D. (2010). Advertising, promotion and integral communication in marketing (Fourth). Mexico: Pearson Prentice Hall.

11. Cuartas, S. A., \& Pérez-Torre, M. É. (2017). The pediatrician as a bioethical link between children's advertising and the family environment. Cuban Journal of Pediatrics., 89 (4), 12.

12. Duan, T., Ding, R., Hou, W., \& Zhang, J. Z. (2018). The burden of attention: CEO publicity and tax avoidance. Journal of Business Research, 87, 90-101. https://doi.org/10.1016/j.jbusres.2018.02.010

13. Ferrer, I., \& Medina, P. (2014). Business management of the Advertising Agency. Madrid, Spain: Diaz de Santos.

14.García-López, J. (2017). Advertising, needs and decrease. Towards a less commercial advertising communication. Chasqui Latin American Journal of Communication, (134), 16.

15. Huacallo-Valdez, L.A. (2018). Business plan for the implementation of a new means of digital advertising in urban transport vehicles in the city of Arequipa. National University of San Agustín, Arequipa, Peru.

16. INEC. (2016). Information Technology and Communications (TIC's) 2016. Retrieved from http://www.eficienterencifras.gob.ec/documentos/webinec/Estadisticas Sociales/TIC/2016/170125.Presentacion Tics 2016.pdf

17. Josemaría, J. A., \& Bedoya, A. M. de. (2017). Communication plan on and off in practice. Madrid, Spain: ESIC Editorial.

18. Kirkpatrick, D. L. (2001). Keys to effective communication. Barcelona, Spain: 2000 Management.

19. Lascano-Cevallos, A. D., Castillo-Pérez, E. I., Mena-Méndez, D., \& Vayas-Ruiz, E. C. (2018). The Feast of fruit and flowers on the local scene of Ambato: analysis of advertising communication (2012-2017). Journal of Urban Studies and Social Sciences, 8 (1), 61-77. 
20. Masaquiza, C., Ballesteros, L., \& González, L. (2017). Stakeholder analysis as input input in the design of an advertising plan. University, Science and Technology, 1, 60-67.

21. Maya, K. (2019). Innovation for the economic development of the Craft Guild. Interdisciplinary Arbitrated Review Koinonía, 4 (7), 88-102. doi: http: //dx.doi.org/10.35381/r.k.v4i7.195

22. O'Guinn, T., Allen, C., \& Semenik, R. (2013). Advertising and Integral Brand Promotion (6th ed.). México, D.F: Cengage Learning Editores.

23. Ortega Polanco, V. (2019). Management of the corporate image of university organizations from the focus of emotional marketing. SCIENCE, 3 (5), 150-171. Recovered

from http://www.cienciamatriarevista.org.ve/index.php/cm/article/view/19

24. Republic of Ecuador Foreign Trade Committee. Resolution No. 050-2014 (2014).

25. Republic of Ecuador Foreign Trade Committee. Resolution No. 011-2015 (2015).

26. Ruíz-Hernández, A. R., Beverido-Sustaeta, P., Salas-García, B., \& Ortiz-León, M. C. (2018). Advertising and smoking of women in Mexico City. Medical Journal of the Universidad Veracruzana, 18 (1).

27. Sainz de Vicuña Ancín, J. M. (2018). The digital marketing plan in practice. ESIC Editorial.

28. Singh, A., \& Duhan, P. (Eds.). (2016). Managing Public Relations and Brand Image through Social Media: https://doi.org/10.4018/978-1-5225-0332-3

29. Tapia, E., \& Castillo, A. (2018). Locals must accept all cards. Retrieved May 26, 2019, from El Comercio website: https://www.elcomercio.com/actualidad/locales-tarcards-credito-debitocomercio.html

30. Vallet, T., Vallet, A., Vallet, I., Casanova, E., Del Corte, V., Estrada, M., \& Monte, P. (2015). Principles of strategic marketing. Castellón de la Plana: Universitat Jaume I. 


\section{CIENCIAMATRIA}

Revista Interdisciplinaria de Humanidades, Educación, Ciencia y Tecnología

Año VI. Vol. VI. Nº10. Enero - Julio 2020

Hecho el depósito de ley: pp201602FA4721

ISSN-L: 2542-3029; ISSN: 2610-802X

Universidad Nacional Experimental Francisco de Miranda (UNEFM). Santa Ana de Coro. Venezuela

Kevin Santiago Lima Haig; Dayra Arelis Segovia Pacheco; César Andrés, Guerrero Velásteguí;

Leonardo G. Ballesteros - López

(02020 por los autores. Este artículo es de acceso abierto y distribuido según los términos y condiciones de la licencia Creative Commons Atribución-NoComercial-Compartirlgual 4.0 Internacional (CC BY-NC-SA 4.0) (https://creativecommons.org/licenses/by-nc-sa/4.0/). 